\title{
Evaluation of Bactigen latex agglutination and Phadebact coagglutination for detection of bacterial antigens in cerebrospinal fluid
}

\author{
YACOOB M COOVADIA, KINNON K NAIDU \\ From the Department of Microbiology, Faculty of Medicine, University of Natal, Durban, South Africa
}

SUMMARY The Bactigen latex agglutination and Phadebact coagglutination tests were evaluated for their ability to detect bacterial antigens of Haemophilus infuenzae type b, Streptococcus pneumoniae (83 serotypes) and Neisseria meningitidis groups A, B, C, and Y in 214 samples of cerebrospinal fluid (CSF). Bactigen latex agglutination was more sensitive than Phadebact coagglutination: it detected $87 \%$ (59/68) of culture positive CSF specimens, whereas Phadebact detected $72 \%(52 / 72)$. Bactigen detected all cases of meningitis caused by $S$ pneumoniae and $H$ infuenzae. Of the 19 specimens that were positive for $N$ meningitidis, $74 \%$ were detected by Phadebact and only $53 \%$ by Bactigen. Gram stain results were positive for $85 \%$ of all specimens positive on culture. Bactigen was slightly more specific (97\%) than Phadebact (96\%). Bactigen, however, showed less specificity $(81 \%)$ than Phadebact $(94 \%)$ on 31 CSF specimens that were culture positive for organisms other than the test organisms. These included two CSF specimens from patients with tuberculous meningitis which gave false positive results for $S$ pneumoniae with the Bactigen reagents. No false positive results were obtained on 104 culture negative CSF samples. Bactigen latex agglutination was superior to Phadebact coagglutination and Gram stain for the detection of $S$ pneumoniae and $H$ infuenzae in CSF specimens from patients with bacteriologically proved meningitis.

In bacterial meningitis rapid and accurate identification of the infecting agent will allow early institution of appropriate antimicrobial treatment. Microscopical examination of Gram stained smears of cerebrospinal fluid (CSF) is the most widely used method for the rapid diagnosis of bacterial meningitis. The Gram stain has certain limitations, however, in that it is reported to have a sensitivity of only $60-70 \%$ for detecting bacteria in CSF.' In addition, the interpretation of results demands appropriate experience in microbiology, which might not always be available especially in small laboratories. Because of these limitations many laboratories now use immunological techniques, which detect bacterial antigens in CSF for the rapid diagnosis of meningitis. These include counterimmunoelectrophoresis, coagglutination, latex agglutination, and enzyme linked immunosorbent assay. The Bactigen latex agglutination, Phadebact coagglutination and BioMérieux latex agglutination CSF kits were

Accepted for publication 18 January 1985 recently introduced in South Africa. In this study, which is part of a continuing study on meningitis, we compared the Phadebact coagglutination and Bactigen latex agglutination techniques for their sensitivity and specificity in detecting bacterial antigens of Streptococcus pneumoniae, Haemophilus infuenzae type b, and Neisseria meningitidis groups A, B, $\mathrm{C}$, and $\mathrm{Y}$ in CSF specimens from patients admitted to hospital with clinical signs and symptoms of meningitis.

\section{Material and methods}

SPECIMENS

CSF specimens submitted to the King Edward VIII Hospital Microbiology Laboratory during the period May to October 1984 were included in the study. Routine investigations performed on all CSF specimens included a differential cell count in a Neubauer counting chamber and biochemical tests for estimation of protein, globulin, chloride and sugar. All specimens were cultured and a Gram stain 
was performed if leucocytes or biochemical abnormalities, or both, were present. If not tested for bacterial antigens immediately, the specimens were stored at $-20^{\circ} \mathrm{C}$ and tested within $24 \mathrm{~h}$.

\section{CULTURES}

The specimens were all cultured on chocolate agar plates, which were incubated at $37^{\circ} \mathrm{C}$ in candle extinction jars. If leucocytes or biochemical abnormalities, or both, were present, additional horse blood agar and MacConkey plates were set up and the CSF also inoculated into supplemented trypticase soy broth (BBL, Maryland, USA). CSF specimens received from patients with clinical diagnoses of tuberculous meningitis were also cultured on to Lowenstein-Jensen media and examined by ZiehlNeelsen stain for acid fast bacilli. All the plates were examined at $24 \mathrm{~h}$ and thereafter daily for a week. The Lowenstein-Jensen bottles were kept for six weeks. All bacterial and fungal isolates were identified by conventional laboratory methods. ${ }^{2}$

\section{BACTIGEN LATEX AGGLUTINATION TEST}

The Bactigen (Wampole Laboratories, Cranbury, USA) $H$ infuenzae type $b, S$ pneumoniae (83 serotypes) and $N$ meningitidis groups $\mathrm{A}, \mathrm{B}, \mathrm{C}$, and $\mathrm{Y}$ latex agglutination tests were performed in accordance with instructions supplied with each kit. Positive and negative controls supplied with the three kits were included in each run. Grossly cloudy or bloody specimens were clarified by centrifugation before testing.

\section{PHADEBACT COAGGLUTINATION TEST}

The Phadebact CSF coagglutination test kit (Pharmacia Diagnostics, Sweden) consists of specific antibodies against $H$ infuenzae type b, $S$ pneumoniae 83 serotypes, $N$ meningitidis groups $\mathrm{A}, \mathrm{B}, \mathrm{C}, \mathrm{Y}$, and $\mathrm{W}$
135 , and streptococcus agalactiae bound to protein A rich staphylococci. The tests were performed according to the manufacturer's instructions. All CSF specimens were heated at $80^{\circ} \mathrm{C}$ for $5 \mathrm{~min}$ before testing. Positive controls, which are available in a separate kit, were included with each run. A built in negative control is provided by the simultaneous use of all four reagents in testing an unknown sample. Results were considered to be non-interpretable if coagglutination occurred at equally strong intensity and speed with more than one reagent.

\section{QUANTITATION}

The strength of agglutination obtained with the Bactigen and Phadebact test kits was quantitated as follows: $1+=$ small but definite clumps against a cloudy background; $2+=$ moderate clumps against a slightly cloudy background; $3+=$ large clumps against a slightly cloudy background; $4+=$ large clumps against a clear background.

\section{SENSITIVITY AND SPECIFICITY}

Sensitivity was determined by the formula: number of true positive results divided by the number of true positive and false negative results. Specificity was determined by the formula: number of true negative results divided by the number of true negative and false positive results.

\section{Results}

Of the 214 specimens included in the study, 79 were culture positive for the test organisms ( $H$ influenzae type b, $S$ pneumoniae, and $N$ meningitidis), 31 were culture positive for other organisms, and 104 were culture negative. Because of the limited amount of CSF available from some patients, it was not always

Comparison of Gram stain, Bactigen latex agglutination, and Phadebact coagglutination for detecting bacterial agents in culture positive CSF specimens

\begin{tabular}{|c|c|c|c|}
\hline \multirow{2}{*}{$\begin{array}{l}\text { Organisms } \\
\text { isolated (total) }\end{array}$} & \multicolumn{3}{|c|}{ No of positive results/no tested $(\%) \ddagger \S$} \\
\hline & Gram & $\begin{array}{l}\text { Bactigen } \\
\text { latex agglutination }\end{array}$ & $\begin{array}{l}\text { Phadebact } \\
\text { coagglutination }\end{array}$ \\
\hline $\begin{array}{l}H \text { infuenzae type b (27)* } \\
S \text { pneumoniae (33)* } \\
N \text { meningitidis (19) } \\
\text { Group B (12) } \\
\text { Group A (1) } \\
\text { Group unknown (6) } \dagger \\
\text { Total (79) }\end{array}$ & $\begin{array}{l}21 / 27(78) \\
30 / 33(91) \\
11 / 13(85) \\
10 / 12(83) \\
1 / 1(100) \\
-\quad 12 / 73(85)\end{array}$ & $\begin{array}{l}21 / 21(100) \\
28 / 28(100) \\
10 / 19(53) \\
7 / 12(58) \\
0 / 1(0) \\
3 / 6(50) \\
59 / 68(87)\end{array}$ & $\begin{array}{l}21 / 24(88) \\
17 / 29(59) \\
14 / 19(74) \\
9 / 12(75) \\
1 / 1(100) \\
4 / 6(67) \\
52 / 72(72)\end{array}$ \\
\hline
\end{tabular}

*A few specimens were tested by Bactigen or Phadebact alone.

tSix specimens received from a peripheral laboratory for which grouping and Gram results were not available.

¥No false positive results obtained by either latex agglutination or coagglutination for 104 culture negative CSF specimens.

\$Six false positive reactions were obtained with Phadebact: five specimens containing $S$ pneumoniae agglutinated with $N$ meningitidis antisera (4) and $H$ infuenzae antisera (1). One specimen containing $H$ infuenzae agglutinated with $S$ pneumoniae antisera. 
possible to test these specimens by both Bactigen and Phadebact. The Table shows the results obtained on CSF specimens that were culture positive for the test organisms. Bactigen was positive for $100 \%$ and Phadebact for $88 \%$ of the $H$ infuenzae b cases of meningitis. Thirty three patients had pneumococcal meningitis. Bactigen detected pneumococcal antigen in all 28 CSF specimens tested, whereas Phadebact was positive for 17 (59\%) of the 29 specimens tested. Of the 13 patients admitted to King Edward VIII Hospital with meningococcal meningitis, 12 harboured group $\mathrm{B}$ and one group A $N$ meningitidis. In addition, six CSF specimens received from a peripheral laboratory and which were culture positive for $N$ meningitidis were also tested. Of these 19 cases of meningococcal meningitis, Phadebact detected $74 \%$ and Bactigen $53 \%$. Follow up, culture negative CSF. specimens from two patients who had been treated for $H$ infuenzae and $S$ pneumoniae meningitis were also tested. These specimens were positive by both Bactigen and Phadebact.

The Phadebact reagents gave false positive results with six CSF specimens that were culture positive for the test organisms. Three specimens containing $S$ pneumoniae gave false positive results with $N$ meningitidis antisera (two specimens) and with $H$ infuenzae antisera (one specimen). In addition, two CSF specimens containing $S$ pneumoniae agglutinated not only with $S$ pneumoniae antisera but also with $N$ meningitidis antisera, and one other specimen containing $H$ infuenzae agglutinated with both $H$ infuenzae and $S$ pneumoniae antisera. In contrast, Bactigen did not give any false positive results with CSF specimens that were culture positive for $S$ pneumoniae, $H$ infuenzae, or $N$ meningitidis.

Thirty one CSF specimens were positive for organisms other than the test organisms and included isolates of Listeria monocytogenes (2), Klebsiella pneumoniae (5), Alcaligenes faecalis (2), Salmonella sp (2), Pseudomonas aeruginosa (2), Acinetobacter anitratus (1), Staphylococcus aureus (1), streptococci (4), Cryptococcus neoformans (5), and Mycobacterium tuberculosis (7). Of these 31 specimens, six (19\%) gave false positive results with Bactigen and two $(6 \%)$ with Phadebact. False positive results for $S$ pneumoniae were obtained with both Bactigen and Phadebact on two CSF specimens containing Alcaligenes faecalis and Pseudomonas aeruginosa. In addition, Bactigen also gave false positive results for $S$ pneumoniae with CSF specimens containing Listeria monocytogenes (1), Klebsiella pneumoniae (1), and Mycobacterium tuberculosis (2). No false positive results were obtained with either Bactigen or Phadebact on 104 culture negative CSF specimens. Non-specific agglutination reactions were noted far more often with Phadebact reagents than with Bactigen, even though all specimens were heated at $80^{\circ} \mathrm{C}$ for 5 min before testing with Phadebact. Gram stain examination of CSF specimens was successful in showing the causative agents in $85 \%$ of cases (Table).

\section{Discussion}

CSF specimens from patients admitted to the paediatric wards of King Edward VIII Hospital, Durban, with clinical and laboratory evidence of bacterial meningitis, often fail to reveal organisms on Gram stain and culture. Many of these patients have received antimicrobial treatment before admission to hospital, but in others there is no obvious reason for these negative findings. The introduction of immunological techniques such as counterimmunoelectrophoresis and passive agglutination has made it possible to detect the causative agents in these cases of partially treated bacterial meningitis. In addition, we envisage using these tests in differentiating partially treated bacterial meningitis from tuberculous meningitis, which in South Africa is still an important cause of meningitis particularly in young black children.

The findings of this study confirm those previously reported that latex agglutination is more sensitive than coagglutination for the detection of bacterial antigens in CSF specimens. ${ }^{34}$ The Bactigen latex agglutination test detected $87 \%$ of all bacteriologically proved cases of meningitis caused by $H$ infuen$z a e, S$ pneumoniae, and $N$ meningitidis compared with $72 \%$ by Phadebact. The sensitivity of detection of $S$ pneumoniae and $H$ infuenzae group b was $100 \%$ by Bactigen compared with $59 \%$ and $88 \%$, respectively, by Phadebact. In accordance with previous findings ${ }^{45}$ we also experienced problems in the detection of $N$ meningitidis antigen. Both Bactigen and Phadebact failed to detect $26 \%$ of all the cases of meningococcal meningitis. Phadebact, however, was more sensitive than Bactigen for the detection of $N$ meningitidis group $b$. This contrasts with the observations of Tilton et al, ${ }^{4}$ who reported detection rates of $66 \%$ by Bactigen (4/6), 50\% by Phadebact $(4 / 8)$, and $80 \%$ by Directigen latex agglutination (4/5). Their results may not be statistically significant, however, because of the limited number of CSF specimens tested. Further studies will need to be undertaken to decide which of the three reagents is the most sensitive for detection of $N$ meningitidis. It should be noted that the Wellcogen and BioMérieux latex agglutination kits do not include reagents for the detection of $N$ meningitidis group $b$, which in our hospital accounts for more than $90 \%$ of all cases of meningococcal meningitis. 
Serological cross reactions among different groups of bacteria in counterimmunoelectrophoresis and agglutination tests have been previously documented. ${ }^{4-8}$ False positive results were noted with Phadebact on $4 \%(8 / 214)$ of CSF specimens examined and with Bactigen on $3 \%(6 / 214)$. With Phadebact false positive results were noted particularly on CSF specimens containing $S$ pneumoniae, which cross reacted with either $N$ meningitidis antisera or $H$ infuenzae group $b$ antisera. In contrast Bactigen gave false positive results with CSF specimens containing organisms other than the test organisms, which cross reacted with $S$ pneumoniae antisera. The false positive reactions obtained with Bactigen $S$ pneumoniae antisera on CSF specimens containing $M$ tuberculosis were particularly disturbing because they could lead to patients receiving inappropriate antimicrobial treatment with potentially serious consequences. These findings emphasise the need for both laboratory staff and clinicians to exercise caution when interpreting results that appear inconsistent with the clinical findings.

Our findings confirm that Bactigen latex agglutination is more sensitive than Phadebact coagglutination for the detection of $H$ infuenzae and $S$ pneumoniae in CSF specimens. Bactigen was also slightly more specific than Phadebact. Because of the subjectivity entailed in reading reactions and the problem of false positive results with both latex agglutination and coagglutination, we would not recommend that these tests be used in isolation for the rapid diagnosis of meningitis. They should always be performed in conjunction with the Gram stain and culture, and where indicated the CSF specimen should also be cultured for $M$ tuberculosis.
We thank Miss Diane Naidu and Mr Leyton Msomi for expert technical assistance. This study was supported in part by a grant from the South African Medical Research Council.

\section{References}

' LaForce FM, Brice JL, Tornabene TG. Diagnosis of bacterial meningitis by gas-liquid chromatography. Il. Analysis of spinal fluid. J Infect Dis 1979;140:453-64.

2 Cowan ST. Cowan and Steel's Manual for the identification of medical bacteria. 2nd ed. Cambridge: Cambridge University Press, 1974:238.

${ }^{3}$ Marcon MJ, Hamoudi AC, Cannon HJ. Comparative laboratory evaluation of three antigen detection methods for diagnosis of Haemophilus infuenzae type b disease. J Clin Microbiol 1984; 19:333-7.

4 Tilton RC, Dias F, Ryan RW. Comparative evaluation of three commercial products and counterimmunoelectrophoresis for the detection of antigens in cerebrospinal fluid. J Clin Microbiol 1984;20:231-4.

s Drow DL, Welch DF, Hensel D, Eisenach K, Long E, Slifkin M. Evaluation of the Phadebact CSF test for detection of the four most common causes of bacterial meningitis. J Clin Microbiol 1983; 18: 1358-61.

- Colding H, Lind I. Counterimmunoelectrophoresis in the diagnosis of bacterial meningitis. J Clin Microbiol 1977;5:405-9.

7 Welch DF, Hensel D. Evaluation of Bactigen and Phadebact for detection of Haemophilus infuenzae type b antigen in cerebrospinal fluid. J Clin Microbiol 1982;16:905-8.

${ }^{8}$ Wasilauskas BL, Hampton KD. Determination of bacterial meningitis: a retrospective study of 80 cerebrospinal fluid specimens evaluated by four in vitro methods. J Clin Microbiol 1982;16:531-5.

Requests for reprints to: Dr YM Coovadia, Department of Microbiology, Faculty of Medicine, PO Box 17039, Congella 4013, Durban, South Africa. 